\title{
Application of the Internet of Things Technology in Smart Grid
}

\author{
Dong Cuiying, Zhou Jianli, Wang Moqi \\ Department of Information, Tangshan college, Tangshan, China
}

\begin{abstract}
According to the application of IOT (Internet of things) in smart grid, intelligent network node of the IOT based on ARM, GSM, computer network and multi sensor technology was designed in this paper. The main function of the node was remote controlling situation of transformation equipment by short message of the mobile, remote transmission equipment on video monitoring through the computer network, carry on the night intrusion alarm through the infrared sensor. In the paper, smart grid node was explained in detail in the working principle, realization of system hardware and software design.
\end{abstract}

KEYWORD: IOT; smart grid; ARM; internet; sensor

\section{INTRODUCTION}

Internet of things (IOT) also called sensor network, refers to a variety of information sensing equipment and devices combined with the Internet, Wireless Network to form a huge network. [1]. At present, multimedia networking technology based on IOT has become a most widely used platform for information exchange. Embedded system is connected with Internet by IOT with Ethernet and Internet technology, so you can easily and inexpensive to transmit information to anywhere in the world and to achieve shared resources.

Smart Grid, that is, through the use of modern digital information technology and automatic control technology, the operating parameters and information of all aspects of the power system can communicate with each other to achieve the reliability of grid operation, security, economy, efficiency and so on[2]. Since the concept of smart grid is proposed, it has been a positive response in the world and the smart grid will be the future of power grid.

Application method of IOT technology in smart grid is to use a variety of sensors and obtain operation parameters and information in power system. Then, the information will be transmitted to the server for computing and processing. That can realize the state monitoring of power system operation to ensure stable and reliable operation of power system.

At present, the development of smart grid and IOT industry will have a great effect on the development of the national economy. How to combine the smart grid and IOT is an important problem to be solved in the development of electric power. In this paper, the IOT technology has applied to the smart grid information communication technology platform. To remote transmission line and transformer, IOT nodes has been designed, thus it ensure the electrical safety.

\section{HIERARCHICAL NETWORK STRUCTURE OF IOT IN SMART GRID}

The IOT architecture in smart grid can be divided into three layers, that is: the perception layer, network layer and application layer, as shown in figure 1.Perception layer collects operation and status information of smart grid; the network layer post all kinds of information that collected by sensing layer to the application layer; application layer analysis and process operation state of smart grid by intelligent computing and data fusion technology.

Smart grid monitoring is mainly composed of remote video monitoring, SMS control and infrared alarm. The working principle is: remote video monitoring Remote video monitoring module capture USB video signals by embedded web server, and then the signals were transmitted through TCP/IP network; the staff use the browser to constitute network video monitoring which work on the production department and transmission department of electricity. 


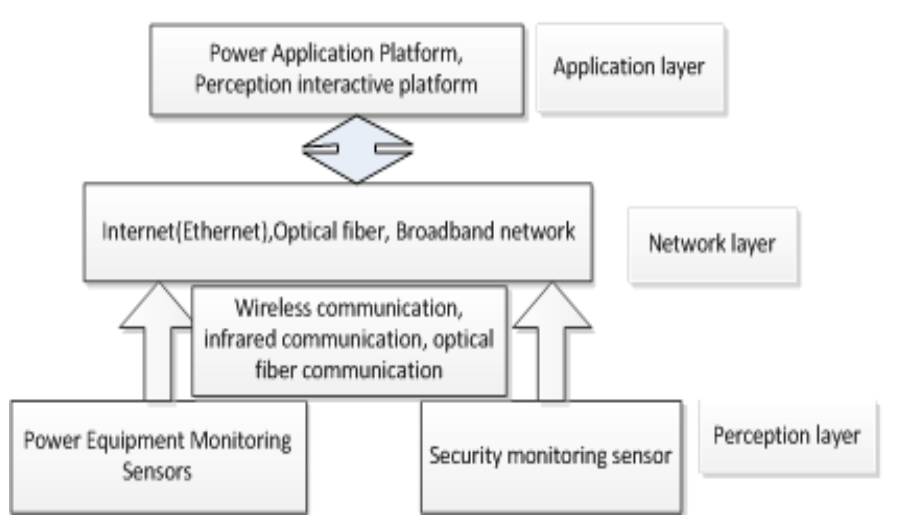

Figure1. Hierarchical network structure of IOT in Smart Grid

In the short message control part, the cell phone can be used to send control messages through the GSM network to the IOT node controller, and then the working states of substation equipment are collected and changed according to the content of the message, and return control results to the monitoring staff by short message. Figure 2 is the schematic diagram of the IOT intelligent controller.

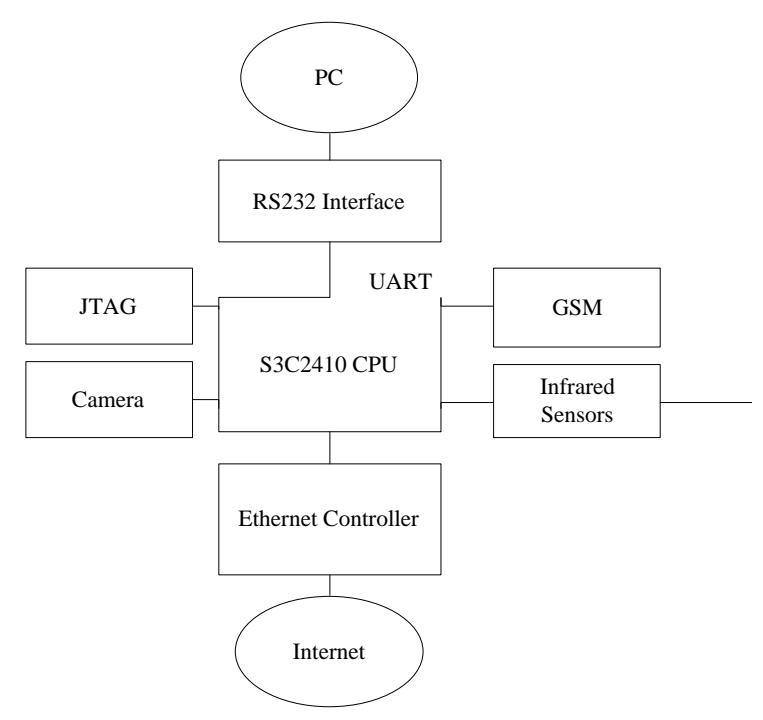

Figure 2. schematic diagram of the IOT intelligent controller

\section{THE HARDWARE DESIGN OF THE SYSTEM CONTROL}

32 bit embedded microprocessor S3C2440 based on ARM920T core is selected as the core of the control system。

The chip integrated including communication memory interface, interface (USB, RS 232), A/D, JTAG, can simplify the peripheral equipment and the hardware connection in system, so the stability and reliability of the system can be improved.

A wireless data transmission module TC35 of SIEMENS company selected as the GSM communication module supports data, short message and voice. TC35 is a new generation of GSM/GPRS dual mode module and is connected with the control center by UART1.

ZC301P chip camera produced by ZTE company was used to capture video and connected with ARM through USB interface. Then the camera stored the video image data collected into buffered. Finally, the image processed was sent to the Internet by a network interface. Infrared sensor connected with ARM by I/O port detects the change of infrared spectrum and sends signals to network node controller for processing.

Text is set in two columns of $9 \mathrm{~cm}$ (3.54") width each with $7 \mathrm{~mm}(0.28 ")$ spacing between the columns. All text should be typed in Times New Roman, 12 pt on 13 pt line spacing except for the paper title (18 pt on $20 \mathrm{pt}$ ), author(s) (14 pt on 16 $\mathrm{pt}$ ), and the small text in tables, captions and references (10 pt on $11 \mathrm{pt})$. All line spacing is exact. Never add any space between lines or paragraphs. When a column has blank lines at the bottom of the page, add space above and below headings (see opposite column).

First lines of paragraphs are indented $5 \mathrm{~mm}\left(0.2^{\prime \prime}\right)$ except for paragraphs after a heading or a blank line (First paragraph tag).

\section{SYSTEM SOFTWARE DESIGN}

Embedded system software consists of three parts, the bootloader, kernel, file system. According to different application, the kernel source code was changed or cut and then the kernel was recompiled [4]. The control core of IOT node controller in this paper is based on the microprocessor ARM, and software of the system include design of system software (embedded operating system, the driver ) and application software.

\subsection{The design of system software}

\subsubsection{Selection and transplantation of embedded operating system}

Linux was used as the operating system, whose version was selected Linux2.6 kernel, and was transplanted in the embedded microprocessor S3C2440.

\subsubsection{The design of the driver}

Many device drivers are needed in the node controller of smart grid networking, but there are few general peripheral drivers can use in embedded systems. In the system, except the GSM module connected with S3C2440 by serial port and can be directly used standard serial port driver, the others as the control interface, the sensor interface and Ethernet interface belonged to non-standard peripherals were needed to design specifically 
drivers. In the design of drivers, the framework of the equipment drivers for embedded Linux system program has a standard, so functions were "filled in" the framework according to the hardware structure[3].

(1)The writing of GSM driver module

static int__init my_init(void)

\{

if(the number of main equipment)

\{

sbc2440_leds_dev $=$ MKDEV (LED_MAJOR,

LED_MINOR);

result=register_chrdev_region

(sbc2440_leds_dev, count, DEVICE_NAME);

\}
else

result=alloc_chrdev_region

(\&sbc2440_leds_dev, LED_MINOR, count,

DEVICE_NAME); \}

LED_MAJOR $=$ MAJOR (sbc2440_leds_dev);

sbc2440_leds_cdev $=$ cdev_alloc( $)$;

if (sbc2440_leds_cdev!= NULL)

\{

cdev_init

(sbc2440_leds_cdev,

\&sbc2440_leds_fops);

sbc2440_leds_cdev->ops = \&sbc2440_leds_fops;

sbc2440_leds_cdev->owner $=$ THIS_MODULE;

if (cdev_add (sbc2440_leds_cdev, sbc2440_leds_dev, count))

printk (KERN_NOTICE "Someting wrong when adding sbc2440_leds_cdev!(n");

else

printk ("Success adding

sbc2440_leds_cdev!(n");

\}

// Create a device file

devfs_handle = devfs_register( NULL,

DEVICE_NAME, DEVFS_FL_DEFAULT,

BUTTON_MAJOR,\&sbc2410_buttons_fops,

NULL);

\}

static int _exit my_exit(void)

\{

// Remove the device file

devfs_remove(DEVICE_NAME);

// Cancel of the character device

cdev_del (sbc2440_leds_cdev);

// Release the device number

unregister_chrdev_region (sbc2440_leds_dev, count);

\}

module_init(my_init );

module_exit( my_exit);

(2)USB camera driver

ZC301P camera was used, so its driver was loaded in the system. Because the Linux kernel does not support this type of camera, so we need load driver module to the kernel. Camera drivers provided by manufacturers were based on the Windows operating system, so we can only choose driver source code that open source in Linux operating system.

The choice of this system is SPCA5XX camera a universal driver. Driver URL in the following: http://mxhaard.free.fr, where we can download driver source code for the embedded environment and there is a special patch for compiling and loading.

As mentioned above, hardware platform, system software and the driver of the system were determined. Next, application of image acquisition software would be developed.

Infrared detection module using general $\mathrm{I} / \mathrm{O}$ port of ARM did not need design special drive.

\subsection{The design of the application}

In smart grid, the role of main program of the IOT node controller was the detection of peripheral and made the control action to various peripherals.

\subsubsection{SMS function sms_send()}

AT commands were used to communicate with TC35 module such as reading and sending SMS. The main use of the AT instruction are as follows

AT+CSCA Set SMS center number ;

AT+CMGF Set the short message mode;

AT+CMGD Delete the message;

AT+CMGS Send message;

AT+CMGR Read the message 。

Messages were sent by the sms_send () function. The AT command "AT+CMGS" wrote data to be transmitted sequentially into the serial port and "ctrl+z" was used as a sign of the end to send text messages.So "ctrl+z" must be sent as a character, and the ASCII code of the "ctrl+z" is 26 .

The following code achieved the design in paper

char ctrlz=26;

sprintf(cmd1,"AT+CMGS=\%d\r",len); //len as the SMS length

write (portfd,cmd1,strlen $(\mathrm{cmd} 1)) ; /$ Writes the command

sleep(1); // one second delay, Waiting serial process was complete

write(portfd,m,strlen(m)); // The content of the message is written

write(portfd,\&ctrlz,1); // Write the ctrl+z as end of SMS and send confirmation

\subsubsection{Transplantation of video server application Servfox}

Download ServfoxRl_0_0.tar.gz from http://mxhaard.free.fr/Spca50x/embedded/Servfox. After decompression, a Makefile.arm file can be 
found in the directory. The compiler can be opened by Vi to modify the path, then modify file to the Makefile[5]. Servfox program can be successfully generated after make.

\section{EXPERIMENTS}

First, bootloader and Linux file system were burned into the development platform in turn; then, compiled applications were added to Linux by the NFS network file ; third, /etc/init.d/rcS file was modified so that the program can run with electric start. RcS file is a script file that can run automatically after the system starts running, which is similar to boot function of the Windows. In this paper, the design of the $\mathrm{rcS}$ file is amended as follows:

\#!/bin/sh

ifconfig eth0 10.10.24.25

mdev $-\mathrm{s}$

boa\&

servfox -d /dev/video0 -s 640x480 -w 7070

gsm\&

Finally the system is powered on after the USB camera and GSM module are plug, then the super terminal output:

servfox version: 1.1.2 date: 07:10:2005 (C) mxhaard@magic.fr

Waiting ....for connection. CTrl_c to stop !!!!

This indicates that the system start complete and wait for a connection, and remote monitoring, SMS remote control and infrared alarm modules have been started successfully at this moment.

In the system, the IP address of video server was entered in the browser address bar of PC within the LAN, then monitoring image collected by camera can be displayed in the Webpage.
When he receives SMS of the transformer 1 failure, the staff will send SMS "open transformer 2". Transformer 2 was start by IOT node controller, thus switch equipment complete.

\section{CONCLUSIONS}

At present, though the power system is gradually to the intelligent direction and the application of IOT in smart grid is still in the primary stage, the Internet of things technology with its interdisciplinary, full range, depth perception "intelligent information sensing peripheral" features have the very good application prospect in the smart grid.

Video monitoring used the IOT technology, switching substation equipment and infrared security will push the development of intelligent in the power grid. IOT node controller with ARM, GSM network, computer network and multi sensor technology has a good prospect and wide application market.

\section{REFERENCES}

[1] Alexandre Santos, Joaquim Macedo, António Costa, M. João Nicolau Internet of Things and Smart Objects for M-health Monitoring and Control Original Research Article, Procedia Technology, 16(2014) 1351-1360.

[2] GONG Gang-jun, SUN Yi, CAI Ming-ming et al. Research of network architecture and implementing scheme for the internet of things towards the smart grid. Power System Protection and Control, 2011, 39(20), P52-58.

[3] Zhou Guangbin Zeng XiaopingThe realization and design of GSM short message intelligence controller. Microcomputer Information, 2007, 23(1),P24-26.

[4] Chen lijun. Analysis of Linux operating system kernel. Beijing, Posts \& Telecom Press, 2005.

[5] Yang Jihua, Yan Guoping. Video capture based on S3C2410 and embedded Linux. Microcontroller \& Embedded System, 2004(11), P69-71. 